\title{
Mechanistic and evolutionary questions about epigenetic conflicts between transposable elements and their plant hosts
}

\author{
Alexandros Bousios ${ }^{1}$ and Brandon S. Gaut ${ }^{2}$ \\ ${ }^{1}$ School of Life Sciences, University of Sussex, Brighton, UK \\ ${ }^{2}$ Department of Ecology and Evolutionary Biology, UC Irvine, Irvine, CA 92697, USA
}

Author for Correspondence:

Brandon S. Gaut; Department of Ecology and Evolutionary Biology, UC Irvine, Irvine, CA 92697, USA; bgaut@uci.edu 


\begin{abstract}
Transposable elements (TEs) constitute the majority of plant genomes, but most are epigenetically inactivated by their host. Research over the last decade has elucidated many of the molecular components that are required for TE silencing. In contrast, the evolutionary dynamics between TEs and silencing pathways are less clear. Here, we discuss current information about these dynamics from both mechanistic and evolutionary perspectives. We highlight new evidence that palindromic sequences within TEs may act as signals for host recognition and that cis-regulatory regions of TEs may be sites of ongoing arms races with host defenses. We also discuss patterns of TE aging after they are silenced; while there is not yet a consensus, it appears that TEs are removed more rapidly near genes, such that older TE insertions tend to be farther from genes. We conclude by discussing the energetic costs for maintaining silencing pathways, which appear to be substantive. The maintenance of silencing pathways across many species suggests that epigenetic emergencies are frequent.
\end{abstract}

\title{
Introduction
}

Transposable elements (TEs) and their plant hosts engage in a continuous battle, whereby TEs seek to proliferate and hosts strive to control their proliferation. In evolutionary terms, it is difficult to declare a winner. On one hand, TEs have been successful by any measure; the majority of plant genomes are composed of TEs that vary from young intact insertions to old fragmented copies. This composition suggests that TEs often overcome 
host defenses, at least on evolutionary timescales. On the other hand, most TEs are epigenetically silenced under normal conditions, so that they do not proliferate. This near-universal suppression implies that plant hosts are largely in control of TE activity. It is the conflicting nature of these two observations that makes the dynamics of host-TE interactions a particularly fascinating topic.

Epigenetic silencing of TEs is mainly conducted via a process known as RNAdirected DNA methylation (RdDM). As its name implies, RdDM uses RNAs, in particular small interfering RNAs (siRNAs), to guide epigenetic modifications that lead to pre-transcriptional silencing of TEs. RdDM has been studied intensively for many years, leading to the elucidation of its mechanisms and pathways. Here, we will not cover the interactions among RdDM components in detail, as these have been presented in several recent reviews [1-3]. Rather, we will first provide an overview of the potential states of a TE within a host genome - and the silencing pathways that influence those states - before highlighting questions of particular interest from mechanistic and evolutionary perspectives.

\section{An overview of the TE life cycle within plant genomes}

We begin by considering an active TE, which is transcribed by RNA polymerase II ( $\mathrm{Pol}$ II) to start its life cycle towards producing new copies (Figure 1). At some point, the endogenous RNAi pathway recognizes these TE mRNAs and degrades them. This process is aided by key factors such as RNA-directed RNA polymerase 6 (RDRO) that converts single-stranded mRNA (ssRNA) into double-stranded RNA (dsRNA), Dicer-like 2 and 4 (DCL2/DCL4) that cleave dsRNA into 21-22nt siRNAs, and Argonaute 1 (AGO1) that 
loads these siRNAs to guide mRNA cleavage (by sequence complementarity between the siRNAs and target mRNAs) [4,5]. This post-transcriptional regulation can degrade large numbers of TE mRNAs, but, most importantly, it may also trigger the "initiation" of pretranscriptional TE silencing, hence providing an entry-point into RdDM (Figure 1).

The transition from post-transcriptional $R N A i$ to pre-transcriptional RdDM was unknown until recently, but there are now two models of how this occurs. The first suggests that some 21-22nt siRNAs are not loaded onto AGO1, which directs mRNA cleavage via $R N A i$, but onto a related factor, $A G O 6$, that guides chromatin modifications [6]. The second proposes that the overproduction of TE mRNAs eventually overwhelms the processing capacity of $D C L 2 / D C L 4$, thereby triggering the biogenesis of $24 \mathrm{nt}$ siRNAs by another Dicer-like protein, $D C L 3$ [7]; the production of $24 \mathrm{nt}$ siRNAs is a hallmark of pre-transcriptional RdDM silencing (Figure 1).

Once RdDM has begun, the "establishment and spreading" of silencing requires two plant-specific RNA polymerases (Pol IV and Pol V). Pol IV transcribes TEs, and the resulting ssRNAs are processed this time by $R D R 2$ and $D C L 3$ to produce $24 \mathrm{nt}$ siRNAs (Figure 1). These 24nt siRNAs are then loaded onto AGO4/AGO6 to direct them to scaffolding, chromatin-bound transcripts of TEs, which are produced by $\mathrm{Pol} V$. The association of the $A G O$ complex with $\mathrm{Pol} V$-derived transcripts mediates cytosine methylation and the deposition of repressive histone marks to the genomic TE locus [13]. Crucially, recent work in Arabidopsis has shown that $P o l I V$ and $P o l V$ are recruited to loci that already contain $\mathrm{H} 3 \mathrm{~K} 9 \mathrm{me}$ and cytosine methylation, respectively $[8,9]$. These observations indicate that $\mathrm{RdDM}$ acts in a self-reinforcing loop that increases the deposition of silencing marks [5], as shown in Figure 1, eventually generating a dense 
heterochromatic environment that restricts access to the Pol II transcriptional machinery. After sufficient deposition, a TE is effectively in a silenced state (Figure 1).

Once silencing is established, repressive chromatin modifications are faithfully copied during DNA replication, enabling inheritance of the silenced chromatin state in daughter cells; this "maintenance" phase of silencing [5] leads to deeply silenced TEs (Figure 1). Overall, these mechanisms appear to be very effective, because only a few TEs have been observed to actively transpose and proliferate, such as the $A c / D s$ and Mutator families of maize and the mPing element in rice [10-12].

Nonetheless, although most TEs are inactive, many may retain the ability to transpose. Genome-wide and biochemical analyses have shown that silenced elements can be activated during epigenetic loss or remodeling (Figure 1), as for example in mutants that interrupt silencing $[13,14]$, in specific cell types like the companion cell of the female gametophyte or the pollen vegetative nucleus $[15,16]$, in developmental transitions [17], and even during stressful conditions [18].

\section{Initiation of silencing}

After articulating the states of the TE life cycle, we now address questions about host-TE interactions, starting by again considering the initiation of silencing. In this context, we first discuss the potential for memory in the host response, before considering how hosts might identify TE mRNAs for post-transcriptional RNAi. We then argue that cisregulatory regions of TEs may be locations of evolutionary conflict with host defenses. Finally, we note the paradox that TEs appear to be quickly resilenced, and yet they are also capable of amplification bursts that produce thousands of genomic copies. 


\section{How efficient is the homology-dependent siRNA surveillance system?}

If a TE has escaped suppression and is reactivated, host defenses must employ intricate ways to reinitiate silencing. As discussed by Fultz et al. [1], one mechanism may be mediated by trans-acting $24 \mathrm{nt}$ siRNAs that have sequence homology to the active TE. Presumably, these 24nt siRNAs are produced from Pol IV-derived transcripts of silenced relatives of the same family, thereby acting as the host's "immune memory" to identify active elements. In support of this model, there is evidence that reactivated TEs in Arabidopsis and maize are silenced in trans by the Pol IV-RdDM pathway [18-20]. The presence or absence of immune memory may also determine whether a horizontal TE invasion will proliferate rapidly in its new host before it is silenced (Figure 1). If, for instance, an endogenous, silenced TE shares substantial sequence homology with an invading TE, then its 24nt siRNAs may constrain the invader's ability to proliferate.

There are, however, indications that this homology-dependent pathway is not always sufficient to silence active TEs. For example, the Evade element of the ATCOPIA93 family in Arabidopsis remains active despite the presence of silenced relatives [21]. Instead, it is suppressed only when enough mRNA transcripts are produced to overwhelm DCL2/DCL4 processing [7]. Also, recent genome-wide studies of LTR retrotransposons $[22,23]$ have shown that certain families, such as $J i$ and Opie in maize, have homology to large numbers of 21-22nt siRNAs, implying that at least some members first undergo post-transcriptional $R N A i$ (Figure 1). If the homology-dependent pathway were able to suppress active TEs quickly, then one would not expect large populations of 21-22nt siRNAs. 


\section{How are TE mRNA transcripts recognized?}

If the homology-dependent pathway fails, silencing of active TEs must be initiated by $R N A i$. The first step of this process likely entails the recognition of Pol II-derived TE mRNAs. Importantly, RNAi needs to safely distinguish between TE and genic mRNAs, but this must be difficult given high sequence and structural diversity among TE classes and, hence, the lack of a universal TE 'barcode'. The recognition of TE mRNAs is mediated by $R D R 6$, which converts ssRNA into dsRNA [3]. It has been hypothesized that $R D R 6$ is assisted by 'primary' small RNAs that act either directly as primers or indirectly by their association with $A G O$ proteins that cleave TE mRNAs, thereby providing a starting point for dsRNA synthesis [24,25]. Indeed, some miRNAs play this role in Arabidopsis [26]. However, miRNAs also participate heavily in genic mRNA cleavage, and so the mechanism of recognizing TE over genic mRNAs remains uncertain. Furthermore, only a subset of Arabidopsis TE families are silenced via miRNAs [26], implying that more mechanisms must be in place.

Intriguingly, one such mechanism could involve non-miRNA hairpin-derived small RNAs (hpRNAs) that are generated from secondary structures within the TE transcripts themselves. The first evidence for the importance of hpRNAs came from the $M u D R$ family in maize. In this example, a spontaneous inverted repeat rearrangement within a single element (termed $M u$ killer) triggered $R N A i$ and subsequent silencing of other $M u D R$ members $[19,27]$. In fact, the discovery of $M u$ killer prompted Lisch and Slotkin to suggest that the low fidelity of TE replication may increase the chances of accidental hairpins to form, which in turn would be a critical feature for enhancing $R N A i$ 
[28]. Axtell further discussed the potential importance of plant hpRNAs and noted that the numerous short hairpins in genomes (which also occur within TEs) may produce hpRNAs [29]. Although hpRNAs have not yet been identified systematically [30], they appear to be abundant in maize [31].

The potential role of hairpins in TE recognition is supported by a new study of maize Sirevirus LTR retrotransposons, which contain multiple palindrome motifs in their LTRs [23]. The palindromes are predicted to form stable stem-loop structures with minimum free energy comparable to those of known miRNA precursors (Figure 2A), raising the possibility that they act as loci for hpRNA biogenesis (Figure 2B). Consistent with this hypothesis, the palindromes are hotspots for siRNA mapping, especially of 2122nt siRNAs [23]. These siRNAs may be directly produced by the hairpins (i.e. without prior dsRNA synthesis by $R D R 6$ ), based on previous research in Arabidopsis showing that $D C L$ proteins can directly process hairpins into hpRNAs [32]. These observations suggest a model in which the palindromes form hairpins in the Sirevirus mRNA, these hairpins are then processed by $D C L$ proteins to produce hpRNAs, and the hpRNAs mediate RDR6 synthesis of dsRNA either as primers or through their loading onto an $A G O$ protein, hence initiating the $R N A i$ cycle. Alternatively, the hairpins could directly prime RDR6 synthesis without the need of hpRNAs, i.e. in a 'primary' small RNAindependent manner [33]. If these models are correct, palindromic regions may be crucial signals for the initiation of TE silencing.

\section{An arms-race between TE cis-regulatory regions and host defenses?}


The Sirevirus palindromes raise an interesting evolutionary question. From the selfish view of a TE, it is not entirely desirable to contain a motif that facilitates host recognition and subsequent silencing. Why, then, might Sireviruses contain such motifs? The answer is simple: because they may be required for the element to proliferate. Indeed, the Sirevirus palindromes are found in the cis-regulatory region in the upstream half of the LTR of retrotransposons [34,35] and retroviruses [36]. Intriguingly, this region in Sireviruses is also an area of rapid sequence evolution, because the palindromes differ along the sequence of an element, among family members, and among families [23]. As a consequence of this extensive sequence variation, each palindrome is targeted by different siRNAs. This example raises the possibility that cis-elements are foci for an evolutionary arms race between TEs and host defenses. Jacobs et al. beautifully describe this interplay for the $L 1$ and $A l u$ TE families in primates [37]. In this case, sequence and structural changes within $L 1$ and $A l u$ regulatory regions led to multiple amplification bursts during primate evolution. These changes, however, were soon followed by changes in the silencing components of the host, leading to cycles of TE proliferation followed by host repression.

Detailed analyses of the epigenetic importance of the cis-regulatory regions of plant TEs are limited, and their potential role in host recognition remains virtually unexplored. However, studies across TE families in diverse organisms (mice, Drosophila, tomato, hominoids) have revealed that cis-regulatory regions are often arranged as arrays of repeats, with some also exhibiting symmetrical properties like those of maize Sireviruses [38-41]. Therefore, the complex, sometimes palindromic organization of regulatory motifs may be common across TE classes. In fact, for the Tnt1 family of 
tomato and other Solanaceae species, the cis-regulatory region encompasses the highest sequence variability among its family members, and this variability associates with different stress-related expression patterns $[42,43]$. These observations suggest that the evolution of this region equipped Tntl subgroups with diverse regulatory capacity to respond to different stimuli and to colonize new hosts $[43,44]$. Similar patterns of diversity within the cis-regulatory region was also recently reported among $T c s$ elements in citrus [45].

Collectively, these observations highlight the need for in-depth research on the evolutionary interplay between cis-regulatory TE motifs, secondary structures and epigenetic silencing. It is possible that these regions evolve quickly to evade the host, but have underlying structural restrictions that continue to facilitate host recognition. For example, at least three kinds of information could support the role of cis-regulatory palindromes in host recognition. First, it must be shown experimentally that these regions are sufficient to generate hpRNAs. Second, their existence needs to be surveyed systematically across TE families and hosts. It is already known that most Sireviruses contain palindromic structures across plant species [46], but it is unclear if other TEs typically have similar structures. Finally, observations about siRNA targeting need to be generalized. Is it common that palindromic regions are hotspots for siRNA mapping? To our knowledge, only the maize Sirevirus study has been performed at a sufficient level of detail to investigate this question.

\section{The paradox of the time to regain control}


There is increasing evidence that epigenetic defenses respond quickly to TE activation, suppressing their activity within only a few host generations. For example, the ONSEN LTR retrotransposon was activated in heat-stressed Arabidopsis seedlings. However, ONSEN only managed to insert new chromosomal copies in the progeny of an siRNAcompromised genetic background and not in the progeny of wild-type plants or in vegetative tissues [18]. Another study in Arabidopsis showed that, after severe loss of methylation, many TEs were remethylated in only a few host generations [20]. Finally, when the Evade element was awakened in recombinant inbred lines engineered to erase most of their methylation, it was transcriptionally silenced after $\sim 15$ host generations, or after $\sim 40$ new copies had been integrated in the genome [7].

Based on these observations, we are faced with a paradox: if TEs are rapidly resilenced, then how do the bursts of amplification occur that ultimately produce thousands of TE copies? Unfortunately, we do not yet have a compelling answer to this question, even though myriad studies have identified evidence for such bursts. For instance, comparative analysis of species from the Oryza genus [47] - as well as work on the $1 \mathrm{~Gb}$ sequence of chromosome $3 \mathrm{~B}$ in wheat $[48,49]$ and the fully sequenced $2.3 \mathrm{~Gb}$ maize genome $[50,51]$ - have documented the accumulation of thousands of copies for several TE families, typically within an estimated timescale of thousands of years. Given that experimental data have demonstrated only small increases in TE copy number, how do bursts of thousands of copies occur? Do TE families escape silencing for longer lengths of time than have been observed experimentally? Or, do they undergo repeated cycles of short bursts that last only a few host generations, producing a few new copies 
before they are resilenced, and then somehow burst again? Thus far, we have very few insights into the timescale and conditions of bursts.

\section{Establishment, Spreading and Maintenance of silencing}

In contrast to the various mysteries associated with the initiation of silencing, the next two phases of host response, that is the RdDM-mediated establishment \& spreading of silencing and the maintenance of repressive chromatin modifications (Figure 1), are much better understood. Yet, it is during these phases that TEs begin to age and gradually degrade. These quiescent TEs shape host genomes substantially, because $i$ ) they are the primary determinants of genome size [52], ii) they may be exapted for regulatory and other purposes [53], and iii) their methylation perturbs gene expression [54]. Given these effects, it is surprising that little is known about the epigenetic profiles of TEs as a function of their age, and how this relationship influences genome structure and function. We address these issues below.

\section{The intriguing epigenetic dynamics of TE ageing}

Only a handful studies have investigated the evolutionary relationship between the age of TEs and either siRNA targeting $[23,54,55]$ or methylation [23,54,56]. These analyses have shown that older elements are typically targeted at lower levels by siRNAs compared to younger elements. This implies that older TEs are likely to be deeply silenced, a state that can be maintained independently of siRNAs (Figure 1). If true, one also expects methylation to increase with age, as elements reach their maximum methylation levels. This expectation holds for LTR retrotransposons in maize [23] and 
even mice [57,58], but not rice [56]. It is not yet clear if the differences between rice and maize are species-specific or specific to the TE families examined.

There are also interesting dynamics among the epigenetic state of a TE, its age, and its proximity to genes. Generally speaking, TEs near genes are thought to be younger than TEs far from genes. There are at least two reasons to expect such a relationship. First, elements near genes may be removed by natural selection more efficiently, because of their deleterious effect on gene expression, which is in turn stronger when a TE is in a silenced state $[54,56,59]$. (We note, however, that the strength of deleterious effects varies among TE families [60]). Second, genes tend to reside in high recombination regions, where natural selection is more efficacious [61] and rates of TE removal are higher [62]. These two factors combine to suggest a model in which TEs are more prone for deletion near genes, such that older elements have a better chance to survive far from genes. To test this model, we retrieved the age for $\sim 6,500$ maize Sireviruses from [63] and examined the age distribution in relation to gene proximity (Figure 3). Insertion age exhibits a positive (but weak) correlation with distance to the nearest gene (Pearson $r=$ $\left.0.1 ; P<10^{-20}\right)$, suggesting that Sireviruses are removed from the genome quicker when they reside near genes.

However, there is no firm agreement on these purported relationships. Recently, Maumus and Quesneville developed a method to estimate the age of elements based on their divergence from a consensus sequence [64]. In contrast, most studies have so far relied on either dating retrotransposons by the divergence between their LTRs, as with the Sirevirus example (Figure 3), or by inferring new vs. old insertions by detecting orthologous loci in other species. Using their age estimates in Arabidopsis, Maumus and 
Quesneville found the exact opposite of previous studies, i.e. that older TEs are both closer to genes and more epigenetically active (i.e. less methylated). It is unclear why these results are so markedly different. One option for resolving this ambiguity may be to survey LTR retrotransposons across several species, because the LTR dating method is generally well accepted. Altogether, these results portray the need for more synthesis of the age, genomic distribution and epigenetic profiles of TEs.

\section{Do zombie TEs exist?}

Careful examination of Figure 3 reveals an anomaly: as Sireviruses age, they tend to be farther from genes, with the exception of the small number of elements in last two categories - i.e., elements estimated to be more than 2.5 million years old. Intriguingly,

these very old elements are distinct for even more reasons [23]. First, they have epigenetic properties similar to those of their youngest counterparts ( $<0.5$ million years), that is somewhat lower cytosine methylation but high levels of siRNA targeting. Furthermore, they tend to $i$ ) have an unexpectedly large number of intact palindromes, ii) often contain complete gag and pol genes (suggesting potential competence) and iii) retain extensive homology with siRNAs that cross-map to much younger elements.

What could explain these observations? Lisch and coauthors have hypothesized that plant genomes contain 'zombie' TEs that are co-opted by the host to trigger both their own silencing and the trans-silencing of active relatives [28,65]. By being beneficial to the host, zombies are predicted to be both evolutionary conserved and epigenetically active, hence resembling loci that produce piwi-interacting RNAs in Drosophila [66]. Also, they would be expected to reside in regions near genes where RdDM is ongoing 
and the chromatin environment remains more permissive [67-70]. Thus, one potential explanation for the discontinuity in Figure 3 is that the very old elements have been coopted by the host to produce siRNAs capable of silencing younger, potentially more dangerous, elements. We note that the existence of zombies is as yet only hypothetical, but some properties of very old Sireviruses may be consistent with this hypothesis.

\section{Escape from silencing}

Finally, despite the post-transcriptional $R N A i$ and pre-transcriptional RdDM silencing pathways, TEs do escape suppression (Figure 1). Nonetheless, although TEs can reactivate during epigenetic loss or remodeling, the question remains as to whether they can themselves avoid host defenses.

\section{Have TEs evolved escape strategies?}

Several mechanisms have been proposed to facilitate escape from silencing. These include recombination between different elements, which generates new variants unrecognizable to host defenses [71,72]. Another way to escape silencing is to switch hosts via horizontal transfer. Invading a naïve genome where prior "immune memory" is absent may provide TEs with a chance to freely proliferate. A growing body of data suggests that these events occur more often than initially thought between vertebrates [73] and between invertebrates [74], but research on plants remains underrepresented [75]. Nonetheless, a recent study investigated the genomes of 40 angiosperms and found evidence for extensive horizontal transfer of LTR retrotransposons [76]. Some of these elements underwent subsequent amplification bursts, suggesting both that they remained 
active after initial colonization and that horizontal transfer may be an important factor in plant genome evolution.

Finally, in a fabulous review, Lisch suggested that TEs might evade suppression by capturing gene fragments as a form of camouflage [65]. In theory, if these TEs are targeted by siRNAs, the same siRNAs could target the 'parent' genes from which the fragments were captured and likely alter their expression. Lisch's hypothesis was based primarily on the observation that Pack-MULE and Helitron TEs systematically acquire exons during transposition [77,78]. However, most research on gene capture by TEs has focused on the evolutionary patterns of selection and possible expression of these captured fragments [78-80], and not on the epigenetic consequences on either the TE or the parent gene. Furthermore, the extent of gene capture by LTR retrotransposons, which make up the majority of plant genomes, has not been examined extensively. Studies on the potential effect of gene capture for TE evasion are urgently needed.

\section{Energetic costs of TE silencing and their evolutionary consequences}

Thus far, we have considered the epigenetic states of TEs and addressed several mechanistic and evolutionary questions that suitably fit under these states (Figure 1). If we now step away from the intricacies of the TE life-cycle, we can point out another evolutionary implication: the host response to TEs is not only complex but also likely to be energetically costly. The energetic costs include the production and activation of multiple polymerases, methylases, protein complexes and siRNAs. In evolutionary terms, it does not pay to maintain an energetically expensive process unless it contributes to 
fitness. But how costly are these host mechanisms and under what conditions would they be maintained?

Let us first consider the maintenance of TE repressive chromatin modifications as a de facto necessity for cell integrity; we assume, therefore, that hosts cannot afford to diminish these processes regardless of energetic costs. Our focus then turns to siRNAs, which appear to be produced abundantly from both pericentromeric heterochromatin and euchromatic chromosomal arms based on sequencing and mapping of Pol IV-derived transcripts and their associated siRNAs in Arabidopsis [81]; in fact, $65 \%$ of $\mathrm{Pol} I \mathrm{~V}$ transcripts mapped to TE loci, compared to $9 \%$ to genes [81]. Surprisingly, few papers have directly calculated the proportion of siRNA libraries that map to TEs, despite abundant information from several plant species (for example, http://mpss.udel.edu/). However, limited evidence in Arabidopsis suggests that siRNAs that map to TEs constitute a substantial proportion of small RNAs [30], and up to $11.3 \%$ of siRNAs map to a set of $\sim 6,500$ maize Sireviruses that constitute only $2.8 \%$ of the genome [23]. Given that Sireviruses totally occupy $\sim 20 \%$ of the maize genome [51], it is likely that a much higher proportion of siRNAs corresponds to them. More detailed calculations across plant species and tissues would be valuable, but the overarching impression is that most siRNAs map to TEs.

This raises the question: why incur the energetic costs to produce siRNAs when most TEs are highly methylated [82-84] and, therefore, presumably deeply silenced? There are at least two potential answers. First, as discussed above, siRNAs - and particularly $24 \mathrm{nt}$ siRNAs - may function as immune memory. Under this scenario, siRNAs act as a buffer against the possibility of TE activity, even though most 
methylation is maintained independently of RdDM in heterochromatic regions [70]. The retention of siRNA surveillance would then be similar to the evolution and retention of acquired immune memory in vertebrates, which is costly but maintained under frequent cycles of reinfection [85]. Consistent with this scenario, some TEs are expressed in mutant backgrounds with impeded 24nt siRNA biogenesis [13,70], indicating that the TEs may be released from "establishment and spreading" rather than a "deeply silenced" state (Figure 1). A second possibility is that siRNA production is necessary for a particular step of the host lifecycle, so that it cannot be lost. The fact that Arabidopsis TEs are epigenetically released in companion cells of the male and female gametophyte and then apparently reprogrammed in the sperm and egg cells is consistent with this assertion $[15,16]$. However, if this were the only necessary function for siRNAs, evolution would be expected to dampen production in non-reproductive tissues, thereby conserving costs.

\section{Conclusions and Future directions}

Our primary interest in this review has been to convey questions about the evolutionary dynamics between TEs and hosts. Some questions were more broad and evolutionary, while others specific and mechanistic. All questions, however, shared a common feature in that their answers are likely to be multifaceted. For example, the evolutionary pressures that maintain the host response system may reflect the importance of $24 \mathrm{nt}$ siRNAs in immune memory, the frequency of ongoing emergencies, and perhaps even essentiality for host reproduction. Similarly, signals for host recognition are likely to vary across TEs classes and also among species. 
This raises an important point: most studies have so far focused on Arabidopsis, with a few on rice and maize as well. Nonetheless, these species are not "normal", because they all have smaller genomes than the angiosperm average [52]. Moreover, Arabidopsis is particularly bizarre because its elements tend to be substantially older than those of its sister species Arabidopsis lyrata [86], suggesting a dearth of transpositions in recent past. It is possible, then, that these model plants are atypical with respect to some features of the host response. We therefore endorse continued epigenetic studies across a wide variety of plant species. A helpful first-step will be to study the evolution of genes known to contribute to epigenetic mechanisms. For example, one recent paper has found that lower plants lack important RdDM-related genes [87], suggesting that host-TE interactions differ substantially across the plant kingdom. Similarly, patterns of DNA methylation vary substantially across plant species [88].

There is also an urgent need for more work on the host response as a function of development, even in model plants. One of the most exciting discoveries of the last decade was the finding that TEs were epigenetically released and then reprogrammed within the male and female gametophyte $[15,16]$. Is this reprogramming a necessary step in the Arabidopsis lifecycle? Is it shared with other plants? If so, how broadly? Such studies are technically difficult, but they will ultimately help illuminate critical features of host-TE interactions.

Finally, we know very little about the focal regions of TE-siRNA interactions, because most studies have focused on mapping siRNAs to exemplar TEs $[6,26,31,67,70,82,89,90]$ instead of intact elements. Unfortunately, the process of building a consensus is likely to omit the regions of interest, i.e. those that evolve rapidly 
due to an ongoing arms race between TEs and hosts. The accurate identification and classification of large numbers of individual TEs remains, however, a challenging task $[91,92]$. Nonetheless, a recent review has stressed the need for fine-scale characterization of plant TEs to properly assess epigenetic dynamics [93], and here we amplify the sentiment for continued bioinformatic and evolutionary analyses of TEs .

\section{Acknowledgements}

We thank Nikos Darzentas and Danelle Seymoor for helpful discussions on the manuscript. $\mathrm{AB}$ is supported by the European Community's Seventh Framework Programme (FP7/2007-2013) under grant agreement no: PIEF-GA-2012-329033. BSG is supported by NSF grant IOS-1542703.

\section{Figure captions}

Figure 1. States of TEs and their interactions with the host's epigenetic silencing pathways. Active TEs may derive from either horizontal transfer or the escape of endogenous TEs from epigenetic suppression. The initiation of suppression may depend on homology-dependent silencing or $R N A i$, which is facilitated by known or yet-to-beidentified triggers. $R N A i$ cleaves TE mRNAs post-transcriptionally (PTS), but also places the first heterochromatic marks on TE insertions, hence initiating pre-transcriptional silencing (TS). After initiation, RdDM strengthens suppression in a self-reinforcing loop for the establishment and spreading of silencing. These silenced TEs may slowly reach their maximum methylation levels while ageing, a process that may be largely maintained independent of RdDM. See text for additional details. 
Figure 2. The epigenetic and sequence patterns of the maize Sirevirus LTRs. (A) Minimum free energy (MFE) of siRNAs from a previously published lead library [89] that mapped along the LTRs of two abundant maize Sirevirus families, Ji and Opie. Mapping of siRNAs and calculation of their MFE was conducted as in [23]. The dotted lines represent the $-40 \mathrm{MFE}$ cutoff of known miRNA precursors [31]. A schematic shows the location of palindromes, which correspond to the hairpin-prone regions of the LTRs. (B) Predicted secondary structure of an Opie LTR. Arrows point to two hairpins formed by palindromes and potential locations for processing by $D C L$ proteins into hpRNAs. Note that longer hairpins may form by the folding of two (or more) pairs of palindromes.

Figure 3. The proximity of Sirevirus elements to maize genes as a function of their insertion age. We retrieved information for 6,456 Sireviruses from MASiVEdb (http://databases.bat.infspire.org/masivedb/) and allocated them into age groups as in [23]. The number at the top of each boxplot indicates the number of elements within each group. Gene information was retrieved from the Filtered Gene Set of the maize B73 RefGen_V2 genome. my, million years.

\section{References and recommended reading}

Papers of particular interest, published within the period of review, have been highlighted as:

* of special interest $[1,6,7,76]$

** of outstanding interest $[23,26,37,67,81]$ 
*1. Fultz D, Choudury SG, Slotkin RK: Silencing of active transposable elements in plants. Current Opinion in Plant Biology 2015, 27:67-76.

This review lucidly summarizes the complex pathways of epigenetic silencing, with an emphasis on the least understood step of initiation of silencing.

2. Matzke MA, Kanno T, Matzke AJM: RNA-Directed DNA Methylation: The Evolution of a Complex Epigenetic Pathway in Flowering Plants. In Annual Review of Plant Biology, Vol 66. Edited by Merchant SS; 2015:243-267. Annual Review of Plant Biology, vol 66.]

3. Matzke MA, Mosher RA: RNA-directed DNA methylation: an epigenetic pathway of increasing complexity. Nature Reviews Genetics 2014, 15:394-408.

4. Nuthikattu S, McCue AD, Panda K, Fultz D, DeFraia C, Thomas EN, Slotkin RK: The Initiation of Epigenetic Silencing of Active Transposable Elements Is Triggered by RDR6 and 21-22 Nucleotide Small Interfering RNAs. Plant Physiology 2013, 162:116-131.

5. Panda K, Slotkin RK: Proposed mechanism for the initiation of transposable element silencing by the RDR6-directed DNA methylation pathway. Plant signaling \& behavior 2013, 8.

*6. McCue AD, Panda K, Nuthikattu S, Choudury SG, Thomas EN, Slotkin RK: ARGONAUTE 6 bridges transposable element mRNA-derived siRNAs to the establishment of DNA methylation. Embo Journal 2015, 34:20-35.

This paper shows how 21-22nt siRNAs, which are produced by cleavage of TE mRNA during $R N A i$, are loaded onto AGO6 and direct chromatin modifications of active TEs. 
*7. Mari-Ordonez A, Marchais A, Etcheverry M, Martin A, Colot V, Voinnet O: Reconstructing de novo silencing of an active plant retrotransposon. Nature Genetics 2013, 45:1029-+.

This study follows the epigenetic re-silencing of an active TE in Arabidopsis. After $\sim 40$ copies and $\sim 15$ host generations, DCL2/DCL4 is unable to process all TE mRNAs, shifting to 24 nt siRNAs production via $D C L 3$.

8. Johnson LM, Du JM, Hale CJ, Bischof S, Feng SH, Chodavarapu RK, Zhong XH, Marson G, Pellegrini M, Segal DJ, et al.: SRA- and SET-domain-containing proteins link RNA polymerase $\mathrm{V}$ occupancy to DNA methylation. Nature 2014, 507:124-+.

9. Law JA, Du JM, Hale CJ, Feng SH, Krajewski K, Palanca AMS, Strahl BD, Patel DJ, Jacobsen SE: Polymerase IV occupancy at RNA-directed DNA methylation sites requires SHH1. Nature 2013, 498:385-+.

10. Naito K, Cho E, Yang G, Campbell MA, Yano K, Okumoto Y, Tanisaka T, Wessler SR: Dramatic amplification of a rice transposable element during recent domestication. Proceedings of the National Academy of Sciences of the United States of America 2006, 103:17620-17625.

11. Vollbrecht E, Duvick J, Schares JP, Ahern KR, Deewatthanawong P, Xu L, Conrad LJ, Kikuchi K, Kubinec TA, Hall BD, et al.: Genome-Wide Distribution of Transposed Dissociation Elements in Maize. Plant Cell 2010, 22:1667-1685.

12. Lisch D: Mutator and MULE Transposons. Microbiology spectrum 2015, 3:MDNA3-0032-2014. 
13. Jia Y, Lisch DR, Ohtsu K, Scanlon MJ, Nettleton D, Schnable PS: Loss of RNADependent RNA Polymerase 2 (RDR2) Function Causes Widespread and Unexpected Changes in the Expression of Transposons, Genes, and 24-nt Small RNAs. Plos Genetics 2009, 5.

14. Tsukahara S, Kobayashi A, Kawabe A, Mathieu O, Miura A, Kakutani T: Bursts of retrotransposition reproduced in Arabidopsis. Nature 2009, 461:423-U125.

15. Ibarra CA, Feng XQ, Schoft VK, Hsieh TF, Uzawa R, Rodrigues JA, Zemach A, Chumak N, Machlicova A, Nishimura T, et al.: Active DNA Demethylation in Plant Companion Cells Reinforces Transposon Methylation in Gametes. Science 2012, 337:1360-1364.

16. Slotkin RK, Vaughn M, Borges F, Tanurdzic M, Becker JD, Feijo JA, Martienssen RA: Epigenetic Reprogramming and Small RNA Silencing of Transposable Elements in Pollen. Cell 2009, 136:461-472.

17. Li H, Freeling M, Lisch D: Epigenetic reprogramming during vegetative phase change in maize. Proceedings of the National Academy of Sciences of the United States of America 2010, 107:22184-22189.

18. Ito H, Gaubert H, Bucher E, Mirouze M, Vaillant I, Paszkowski J: An siRNA pathway prevents transgenerational retrotransposition in plants subjected to stress. Nature 2011, 472:115-U151.

19. Slotkin RK, Freeling M, Lisch D: Heritable transposon silencing initiated by a naturally occurring transposon inverted duplication. Nature Genetics 2005, 37:641-644. 
20. Teixeira FK, Heredia F, Sarazin A, Roudier F, Boccara M, Ciaudo C, Cruaud C, Poulain J, Berdasco M, Fraga MF, et al.: A Role for RNAi in the Selective Correction of DNA Methylation Defects. Science 2009, 323:1600-1604.

21. Mirouze M, Reinders J, Bucher E, Nishimura T, Schneeberger K, Ossowski S, Cao J, Weigel D, Paszkowski J, Mathieu O: Selective epigenetic control of retrotransposition in Arabidopsis. Nature 2009, 461:427-U130.

22. Barber WT, Zhang W, Win H, Varala KK, Dorweiler JE, Hudson ME, Moose SP: Repeat associated small RNAs vary among parents and following hybridization in maize. Proceedings of the National Academy of Sciences of the United States of America 2012, 109:10444-10449.

**23. Bousios A, Diez CM, Takuno S, Bystry V, Darzentas N, Gaut BS: A role for palindromic structures in the cis-region of maize Sirevirus LTRs in transposable element evolution and host epigenetic response. Genome Research 2016, 26(2):226-37.

This study maps siRNAs to a large number of LTR retrotransposons in maize, showing that palindromic cis-regulatory regions are hotspots for epigenetic interactions and evolutionary change.

24. Bologna NG, Voinnet O: The Diversity, Biogenesis, and Activities of Endogenous Silencing Small RNAs in Arabidopsis. Annual Review of Plant Biology, Vol 65 2014, 65:473-503.

25. Voinnet O: Use, tolerance and avoidance of amplified RNA silencing by plants. Trends in Plant Science 2008, 13:317-328. 
**26. Creasey KM, Zhai J, Borges F, Van Ex F, Regulski M, Meyers BC, Martienssen RA: miRNAs trigger widespread epigenetically activated siRNAs from transposons in Arabidopsis. Nature 2014, 508:411-+.

This publication demonstrates that miRNAs can target mRNAs of some TE families both for post-transcriptional suppression and, more importantly, for initiation of pretranscriptional silencing.

27. Slotkin RK, Freeling M, Lisch D: Mu killer causes the heritable inactivation of the Mutator family of transposable elements in Zea mays. Genetics 2003, 165:781-797.

28. Lisch D, Slotkin RK: Strategies for silencing and escape: the ancient struggle between transposable elements and their hosts. In International Review of Cell and Molecular Biology, Vol 292. Edited by Jeon KW; 2011:119-152. International Review of Cell and Molecular Biology, vol 292.]

29. Axtell MJ: Classification and Comparison of Small RNAs from Plants. Anпиal Review of Plant Biology, Vol 64 2013, 64:137-159.

30. Coruh C, Shahid S, Axtell MJ: Seeing the forest for the trees: annotating small RNA producing genes in plants. Current Opinion in Plant Biology 2014, 18:8795.

31. Wang X, Elling AA, Li X, Li N, Peng Z, He G, Sun H, Qi Y, Liu XS, Deng XW: Genome-Wide and Organ-Specific Landscapes of Epigenetic Modifications and Their Relationships to mRNA and Small RNA Transcriptomes in Maize. Plant Cell 2009, 21:1053-1069. 
32. Dunoyer P, Brosnan CA, Schott G, Wang Y, Jay F, Alioua A, Himber C, Voinnet O: An endogenous, systemic RNAi pathway in plants. Embo Journal 2010, 29:1699-1712.

33. Devert A, Fabre N, Floris M, Canard B, Robaglia C, Crete P: Primer-Dependent and Primer-Independent Initiation of Double Stranded RNA Synthesis by Purified Arabidopsis RNA-Dependent RNA Polymerases RDR2 and RDR6. Plos One 2015, 10.

34. Grandbastien M-A: LTR retrotransposons, handy hitchhikers of plant regulation and stress response. Biochimica Et Biophysica Acta-Gene Regulatory Mechanisms 2015, 1849:403-416.

35. Kumar A, Bennetzen JL: Plant retrotransposons. Annual Review of Genetics 1999, 33:479-532.

36. Mergia A, Prattlowe E, Shaw KES, Renshawgegg LW, Luciw PA: Cis-acting regulatory regions in the long terminal repeat of Simian Foamy virus type-1. Journal of Virology 1992, 66:251-257.

**37. Jacobs FMJ, Greenberg D, Nguyen N, Haeussler M, Ewing AD, Katzman S, Paten B, Salama SR, Haussler D: An evolutionary arms race between KRAB zincfinger genes ZNF91/93 and SVA/L1 retrotransposons. Nature 2014, 516:242+ .

This publication describes the evolution of the cis-regulatory regions in the $L 1$ and Alu families during primate evolution. Their evolution was met by compensatory changes in the zinc-finger host proteins that are thought to bind to them and mediate their silencing. 
38. Araujo PG, Casacuberta JM, Costa APP, Hashimoto RY, Grandbastien MA, Van Sluys MA: Retrolyc1 subfamilies defined by different U3 LTR regulatory regions in the Lycopersicon genus. Molecular Genetics and Genomics 2001, 266:35-41.

39. Fablet M, Rebollo R, Biemont C, Vieira C: The evolution of retrotransposon regulatory regions and its consequences on the Drosophila melanogaster and Homo sapiens host genomes. Gene 2007, 390:84-91.

40. Ianc B, Ochis C, Persch R, Popescu O, Damert A: Hominoid Composite Non-LTR Retrotransposons-Variety, Assembly, Evolution, and Structural Determinants of Mobilization. Molecular Biology and Evolution 2014, 31:28472864.

41. McDonald JF, Matyunina LV, Wilson S, Jordan IK, Bowen NJ, Miller WJ: LTR retrotransposons and the evolution of eukaryotic enhancers. Genetica (Dordrecht) 1997, 100:3-13.

42. Beguiristain T, Grandbastien MA, Puigdomenech P, Casacuberta JM: Three Tnt1 subfamilies show different stress-associated patterns of expression in tobacco. Consequences for retrotransposon control and evolution in plants. Plant Physiology 2001, 127:212-221.

43. Vernhettes S, Grandbastien MA, Casacuberta JM: The evolutionary analysis of the Tnt1 retrotransposon in Nicotiana species reveals the high variability of its regulatory sequences. Molecular Biology and Evolution 1998, 15:827-836. 
44. Manetti ME, Rossi M, Costa APP, Clausen AM, Van Sluys M-A: Radiation of the Tnt1 retrotransposon superfamily in three Solanaceae genera. $B m c$ Evolutionary Biology 2007, 7.

45. Butelli E, Licciardello C, Zhang Y, Liu J, Mackay S, Bailey P, Reforgiato-Recupero G, Martin C: Retrotransposons Control Fruit-Specific, Cold-Dependent Accumulation of Anthocyanins in Blood Oranges. Plant Cell 2012, 24:12421255.

46. Bousios A, Darzentas N, Tsaftaris A, Pearce SR: Highly conserved motifs in noncoding regions of Sirevirus retrotransposons: the key for their pattern of distribution within and across plants? Bmc Genomics 2010, 11.

47. Piegu B, Guyot R, Picault N, Roulin A, Saniyal A, Kim H, Collura K, Brar DS, Jackson S, Wing RA, et al.: Doubling genome size without polyploidization: Dynamics of retrotransposition-driven genomic expansions in Oryza australiensis, a wild relative of rice. Genome Research 2006, 16:1262-1269.

48. Choulet F, Alberti A, Theil S, Glover N, Barbe V, Daron J, Pingault L, Sourdille P, Couloux A, Paux E, et al.: Structural and functional partitioning of bread wheat chromosome 3B. Science 2014, 345.

49. Daron J, Glover N, Pingault L, Theil S, Jamilloux V, Paux E, Barbe V, Mangenot S, Alberti A, Wincker $\mathrm{P}$, et al.: Organization and evolution of transposable elements along the bread wheat chromosome 3B. Genome Biology 2014, 15.

50. Baucom RS, Estill JC, Chaparro C, Upshaw N, Jogi A, Deragon JM, Westerman RP, SanMiguel PJ, Bennetzen JL: Exceptional Diversity, Non-Random 
Distribution, and Rapid Evolution of Retroelements in the B73 Maize Genome. Plos Genetics 2009, 5.

51. Bousios A, Kourmpetis YAI, Pavlidis P, Minga E, Tsaftaris A, Darzentas N: The turbulent life of Sirevirus retrotransposons and the evolution of the maize genome: more than ten thousand elements tell the story. Plant Journal 2012, 69:475-488.

52. Tenaillon MI, Hollister JD, Gaut BS: A triptych of the evolution of plant transposable elements. Trends in Plant Science 2010, 15:471-478.

53. Feschotte C: Opinion - Transposable elements and the evolution of regulatory networks. Nature Reviews Genetics 2008, 9:397-405.

54. Hollister JD, Gaut BS: Epigenetic silencing of transposable elements: A trade-off between reduced transposition and deleterious effects on neighboring gene expression. Genome Research 2009, 19:1419-1428.

55. Hollister JD, Smith LM, Guo Y-L, Ott F, Weigel D, Gaut BS: Transposable elements and small RNAs contribute to gene expression divergence between Arabidopsis thaliana and Arabidopsis lyrata. Proceedings of the National Academy of Sciences of the United States of America 2011, 108:2322-2327.

56. vonHoldt BM, Takuno S, Gaut BS: Recent Retrotransposon Insertions Are Methylated and Phylogenetically Clustered in Japonica Rice (Oryza sativa spp. japonica). Molecular Biology and Evolution 2012, 29:3193-3203.

57. Faulk C, Barks A, Dolinoy DC: Phylogenetic and DNA methylation analysis reveal novel regions of variable methylation in the mouse IAP class of transposons. Bmc Genomics 2013, 14. 
58. Reiss D, Zhang Y, Rouhi A, Reuter M, Mager DL: Variable DNA methylation of transposable elements The case study of mouse Early Transposons. Epigenetics 2010, 5:68-79.

59. Wang X, Weigel D, Smith LM: Transposon Variants and Their Effects on Gene Expression in Arabidopsis. Plos Genetics 2013, 9.

60. Eichten SR, Ellis NA, Makarevitch I, Yeh CT, Gent JI, Guo L, McGinnis KM, Zhang XY, Schnable PS, Vaughn MW, et al.: Spreading of Heterochromatin Is Limited to Specific Families of Maize Retrotransposons. Plos Genetics 2012, 8.

61. Hill WG, A. R: EFFECT OF LINKAGE ON LIMITS TO ARTIFICIAL SELECTION. Genetical Research 1966, 8:269:268.

62. Ma JX, Bennetzen JL: Recombination, rearrangement, reshuffling, and divergence in a centromeric region of rice. Proceedings of the National Academy of Sciences of the United States of America 2006, 103:383-388.

63. Bousios A, Minga E, Kalitsou N, Pantermali M, Tsaballa A, Darzentas N: MASiVEdb: the Sirevirus Plant Retrotransposon Database. Bmc Genomics 2012, 13.

64. Maumus F, Quesneville H: Ancestral repeats have shaped epigenome and genome composition for millions of years in Arabidopsis thaliana. Nature Communications 2014, 5.

65. Lisch D: Epigenetic Regulation of Transposable Elements in Plants. In Anпиal Review of Plant Biology. Edited by; 2009:43-66. Annual Review of Plant Biology, vol 60.] 
66. Brennecke J, Aravin AA, Stark A, Dus M, Kellis M, Sachidanandam R, Hannon GJ: Discrete small RNA-generating loci as master regulators of transposon activity in Drosophila. Cell 2007, 128:1089-1103.

**67. Gent JI, Ellis NA, Guo L, Harkess AE, Yao Y, Zhang X, Dawe RK: CHH islands: de novo DNA methylation in near-gene chromatin regulation in maize. Genome Research 2013, 23:628-637.

This study reveals that maintenance of TE silencing in heterochromatic regions is based on the methylation factor $D D M 1$ and histone $H 1$ but is largely independent of siRNAs. It also shows that RdDM is responsible for TE silencing near genic regions.

68. Gent JI, Madzima TF, Bader R, Kent MR, Zhang X, Stam M, McGinnis KM, Dawe RK: Accessible DNA and Relative Depletion of H3K9me2 at Maize Loci Undergoing RNA-Directed DNA Methylation. Plant Cell 2014, 26:4903-4917.

69. Sequeira-Mendes J, Aragueez I, Peiro R, Mendez-Giraldez R, Zhang X, Jacobsen SE, Bastolla U, Gutierrez C: The Functional Topography of the Arabidopsis Genome Is Organized in a Reduced Number of Linear Motifs of Chromatin States. Plant Cell 2014, 26:2351-2366.

70. Zemach A, Kim MY, Hsieh P-H, Coleman-Derr D, Eshed-Williams L, Thao K, Harmer SL, Zilberman D: The Arabidopsis Nucleosome Remodeler DDM1 Allows DNA Methyltransferases to Access H1-Containing Heterochromatin. Cell 2013, 153:193-205.

71. Du J, Tian Z, Bowen NJ, Schmutz J, Shoemaker RC, Ma J: Bifurcation and Enhancement of Autonomous-Nonautonomous Retrotransposon Partnership through LTR Swapping in Soybean. Plant Cell 2010, 22:48-61. 
72. Sharma A, Schneider KL, Presting GG: Sustained retrotransposition is mediated by nucleotide deletions and interelement recombinations. Proceedings of the National Academy of Sciences of the United States of America 2008, 105:1547015474.

73. Walsh AM, Kortschak RD, Gardner MG, Bertozzi T, Adelson DL: Widespread horizontal transfer of retrotransposons. Proceedings of the National Academy of Sciences of the United States of America 2013, 110:1012-1016.

74. Bartolome C, Bello X, Maside X: Widespread evidence for horizontal transfer of transposable elements across Drosophila genomes. Genome Biology 2009, 10.

75. Wallau GL, Ortiz MF, Silva Loreto EL: Horizontal Transposon Transfer in Eukarya: Detection, Bias, and Perspectives. Genome Biology and Evolution 2012, 4:801-811.

*76. El Baidouri M, Carpentier MC, Cooke R, Gao DY, Lasserre E, Llauro C, Mirouze M, Picault N, Jackson SA, Panaud O: Widespread and frequent horizontal transfers of transposable elements in plants. Genome Research 2014, 24:831838.

This publication provides evidence for horizontal transfer of TEs across several plant genomes.

77. Du C, Fefelova N, Caronna J, He L, Dooner HK: The polychromatic Helitron landscape of the maize genome. Proceedings of the National Academy of Sciences of the United States of America 2009, 106:19916-19921. 
78. Juretic N, Hoen DR, Huynh ML, Harrison PM, Bureau TE: The evolutionary fate of MULE-mediated duplications of host gene fragments in rice. Genome Research 2005, 15:1292-1297.

79. Barbaglia AM, Klusman KM, Higgins J, Shaw JR, Hannah LC, Lal SK: Gene Capture by Helitron Transposons Reshuffles the Transcriptome of Maize. Genetics 2012, 190:965-975.

80. Yang LX, Bennetzen JL: Distribution, diversity, evolution, and survival of Helitrons in the maize genome. Proceedings of the National Academy of Sciences of the United States of America 2009, 106:19922-19927.

**81. Li S, Vandivier LE, Tu B, Gao L, Won SY, Li S, Zheng B, Gregory BD, Chen X: Detection of Pol IV/RDR2-dependent transcripts at the genomic scale in Arabidopsis reveals features and regulation of siRNA biogenesis. Genome Research 2015, 25:235-245.

This paper identifies the genome-wide locations of Pol IVIRDR2 transcripts and their associated siRNAs.

82. Feng SH, Cokus SJ, Zhang XY, Chen PY, Bostick M, Goll MG, Hetzel J, Jain J, Strauss SH, Halpern ME, et al.: Conservation and divergence of methylation patterning in plants and animals. Proceedings of the National Academy of Sciences of the United States of America 2010, 107:8689-8694.

83. West PT, Li Q, Ji L, Eichten SR, Song J, Vaughn MW, Schmitz RJ, Springer NM: Genomic Distribution of H3K9me2 and DNA Methylation in a Maize Genome. Plos One 2014, 9. 
84. Zemach A, McDaniel IE, Silva P, Zilberman D: Genome-Wide Evolutionary Analysis of Eukaryotic DNA Methylation. Science 2010, 328:916-919.

85. Best A, Hoyle A: The evolution of costly acquired immune memory. Ecology and Evolution 2013, 3:2223-2232.

86. Hu TT, Pattyn P, Bakker EG, Cao J, Cheng JF, Clark RM, Fahlgren N, Fawcett JA, Grimwood J, Gundlach H, et al.: The Arabidopsis lyrata genome sequence and the basis of rapid genome size change. Nature Genetics 2011, 43:476-+.

87. Ma L, Hatlen A, Kelly LJ, Becher H, Wang W, Kovarik A, Leitch IJ, Leitch AR: Angiosperms Are Unique among Land Plant Lineages in the Occurrence of Key Genes in the RNA-Directed DNA Methylation (RdDM) Pathway. Genome biology and evolution 2015, 7:2648-2662.

88. Takuno S, Ran J-H, Gaut BS: Evolutionary patterns of genic DNA methylation vary across land plants. Nature Plants 2016, 2.

89. Diez CM, Meca E, Tenaillon MI, Gaut BS: Three Groups of Transposable Elements with Contrasting Copy Number Dynamics and Host Responses in the Maize (Zea mays ssp mays) Genome. Plos Genetics 2014, 10.

90. Regulski M, Lu Z, Kendall J, Donoghue MTA, Reinders J, Llaca V, Deschamps S, Smith A, Levy D, McCombie WR, et al.: The maize methylome influences mRNA splice sites and reveals widespread paramutation-like switches guided by small RNA. Genome Research 2013, 23:1651-1662.

91. Bousios A, Darzentas N: Sirevirus LTR retrotransposons: phylogenetic misconceptions in the plant world. Mobile DNA 2013, 4. 
92. Hoen DR, Hickey G, Bourque G, Casacuberta J, Cordaux R, Feschotte C, FistonLavier AS, Hua-Van A, Hubley R, Kapusta A, et al.: A call for benchmarking transposable element annotation methods. Mobile DNA 2015, 6.

93. Ragupathy R, You FM, Cloutier S: Arguments for standardizing transposable element annotation in plant genomes. Trends in Plant Science 2013, 18:367376. 


\section{horizontal transfer}
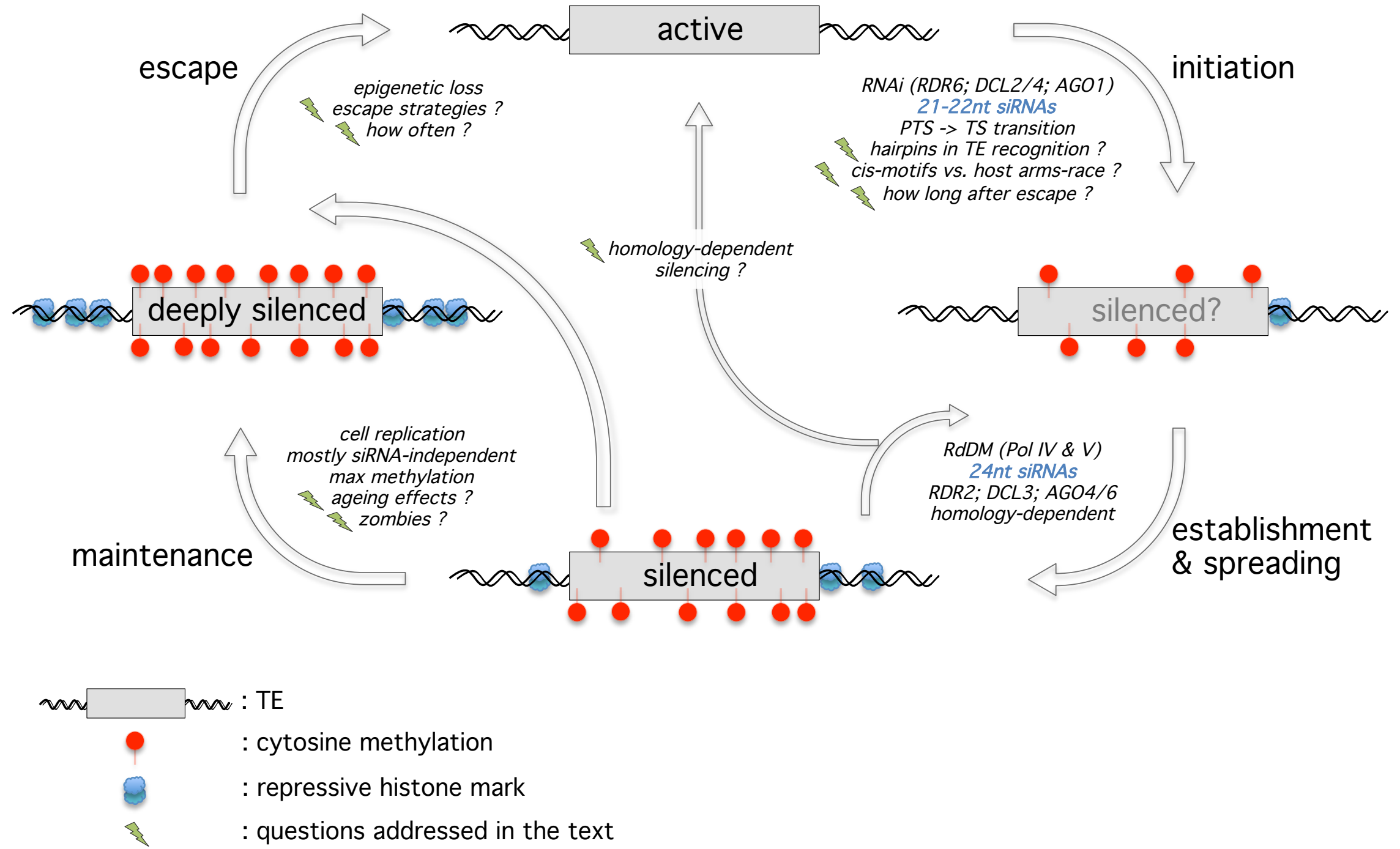


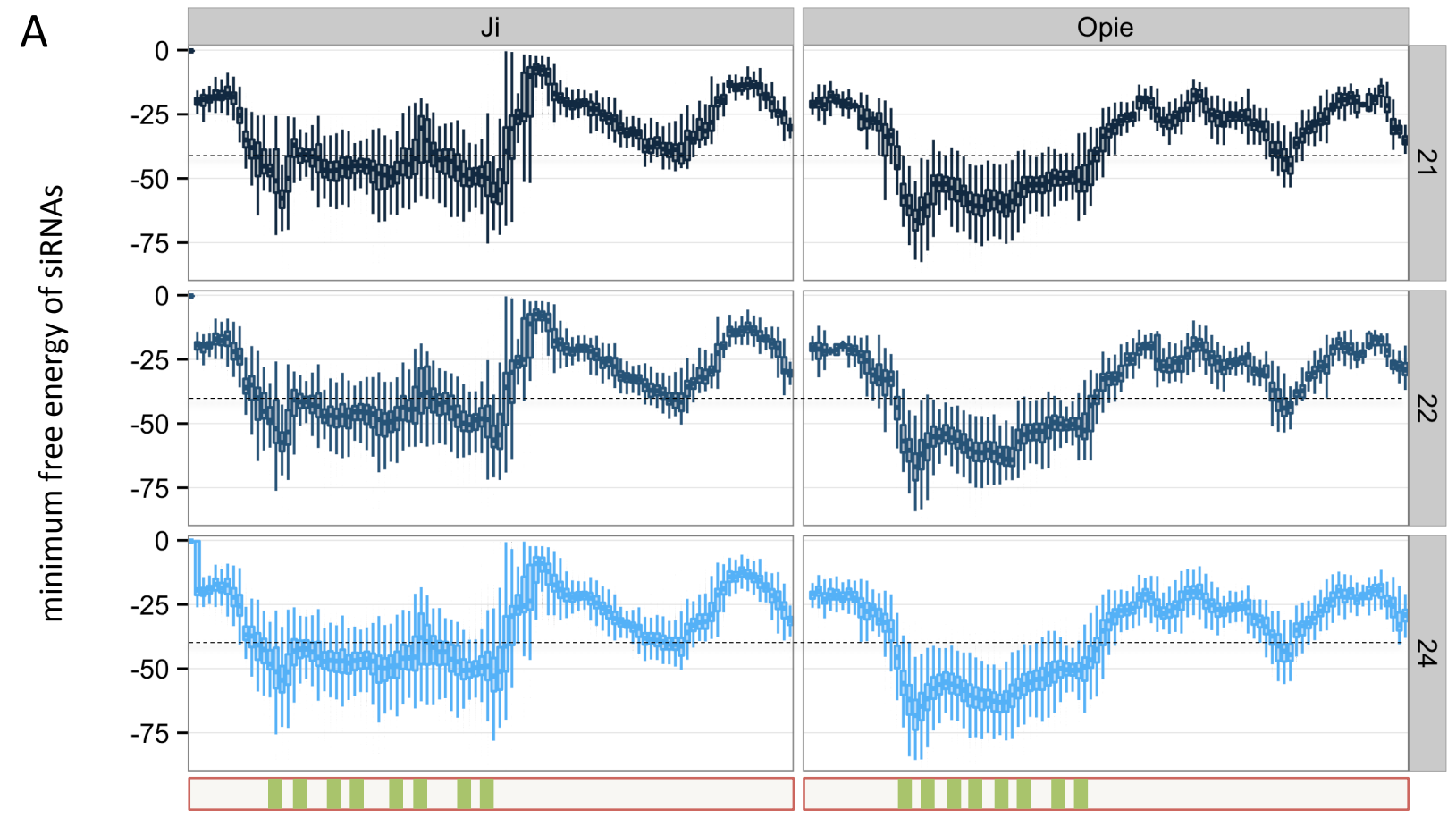

: palindromes

B

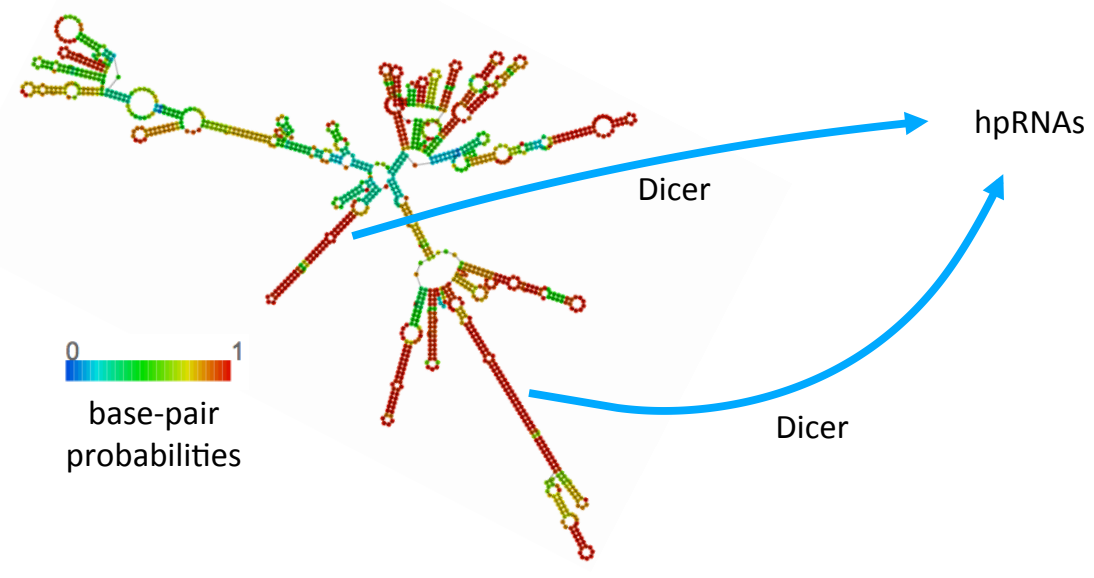




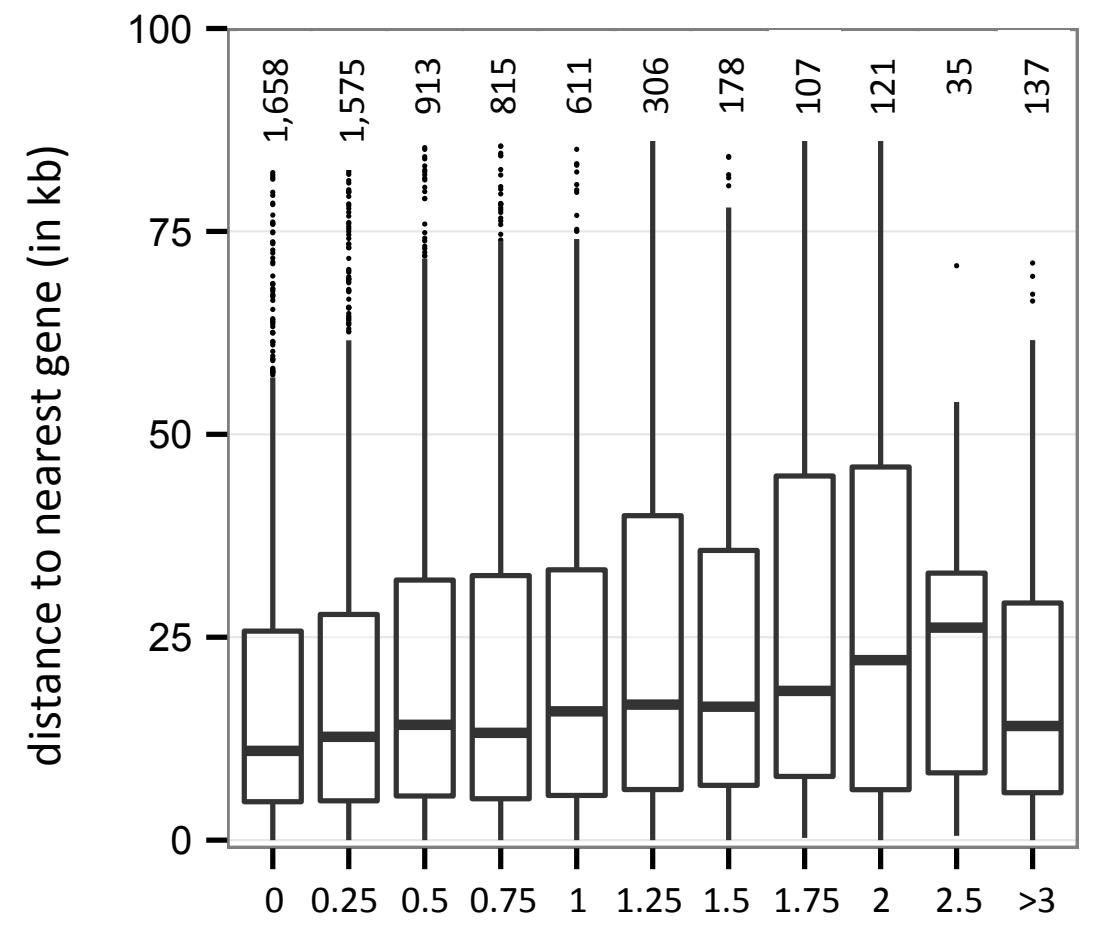

age groups (my) 